\title{
ON THE REGULARITY OF THE HANKEL DETERMINANT SEQUENCE OF THE CHARACTERISTIC SEQUENCE OF POWERS
}

\author{
YINGJUN GUO
}

\begin{abstract}
For any sequences $\mathbf{u}=\{u(n)\}_{n \geq 0}, \mathbf{v}=\{v(n)\}_{n \geq 0}$, we define $\mathbf{u v}:=\{u(n) v(n)\}_{n \geq 0}$ and $\mathbf{u}+\mathbf{v}:=\{u(n)+v(n)\}_{n \geq 0}$. Let $f_{i}(x)(0 \leq i<k)$ be sequence polynomials whose coefficients are integer sequences. We say an integer sequence $\mathbf{u}=\{u(n)\}_{n \geq 0}$ is a polynomial generated sequence if

$\{u(k n+i)\}_{n \geq 0}=f_{i}(\mathbf{u}),(0 \leq i<k)$.

In this paper, we study the polynomial generated sequences. Assume $k \geq 2$ and $f_{i}(x)=\mathbf{a}_{i} x+\mathbf{b}_{i}(0 \leq$ $i<k$ ). If $\mathbf{a}_{i}$ are $k$-automatic and $\mathbf{b}_{i}$ are $k$-regular for $0 \leq i<k$, then we prove that the corresponding polynomial generated sequences are $k$-regular. As a application, we prove that the Hankel determinant sequence $\left\{\operatorname{det}(p(i+j))_{i, j=0}^{n-1}\right\}_{n \geq 0}$ is 2-regular, where $\{p(n)\}_{n \geq 0}=0110100010000 \cdots$ is the characteristic sequence of powers 2. Moreover, we give a answer of Cigler's conjecture about the Hankel determinants.
\end{abstract}

\section{INTRODUCTION}

To introduce our motivation for the problem in this paper, we first recall some basic definitions of automatic and regular sequences.

1.1. Automatic and regular sequences. We say a sequence $\mathbf{u}=\{u(n)\}_{n \geq 0}$ with values in a finite set $k$-automatic if, informally speaking, $u(n)$ is a finite-state function of the base- $k$ expansion of $n$ [10, 3]. This is equivalent to the fact that the $k$-kernel $\mathcal{K}_{k}(\mathbf{u})$ is a finite set [9, 12], where the $k$-kernel is a collection of subsequences

$$
\mathcal{K}_{k}(\mathbf{u})=\left\{\left\{u\left(k^{i} n+j\right)\right\}_{n \geq 0}: i \geq 0,0 \leq j<k^{i}\right\} .
$$

While all $k$-automatic sequences are defined over finite alphabets, Allouche and Shallit [1, 2] introduced a wider class of $k$-regular sequences that are allowed to take values in a Noetherian ring $R$. A sequence is $k$-regular if the module generated by its $k$-kernel is finitely generated. In this paper, unless otherwise stated, the sequences we considered are integer sequences and assume the underlying ring is $\mathbb{Z}$. More precisely, we say that an integer sequence $\{u(n)\}_{n \geq 0}$ is $k$-regular if every sequence of its $k$-kernel is a $\mathbb{Z}$-linear combination of a finite set. That is to say, there exist a finite number of integer sequences $\left\{a_{1}(n)\right\}_{n \geq 0},\left\{a_{2}(n)\right\}_{n \geq 0}, \cdots,\left\{a_{N}(n)\right\}_{n \geq 0}$ such that for any $i \geq 0,0 \leq j<k^{i}$, there exist $c_{1}, c_{2}, \cdots, c_{N} \in$ $\mathbb{Z}$ such that

$$
u\left(k^{i} n+j\right)=\sum_{\ell=1}^{N} c_{\ell} a_{\ell}(n),(n \geq 0) .
$$

The $k$-regular sequences play the same role for integer valued sequences as the $k$-automatic sequences play for sequences over a finite alphabet. More relations between the $k$-regular sequences and the $k$ automatic sequences can be found in [1, 2, 11.

1.2. Polynomial generated sequences. For any sequences $\mathbf{u}=\{u(n)\}_{n \geq 0}$ and $\mathbf{v}=\{v(n)\}_{n \geq 0}$. We define addition and multiplication as follows:

- $\mathbf{u}+\mathbf{v}:=\{u(n)+v(n)\}_{n \geq 0}$,

- $\mathbf{u} \cdot \mathbf{v}:=\{u(n) v(n)\}_{n \geq 0}$.

Let $\mathcal{R}$ denote the set of all integer sequences. Then $(\mathcal{R},+, \cdot)$ forms a commutative ring. Similarly, $\mathcal{R}[X]$, the set of polynomials in the indeterminate $x$ over $\mathcal{R}$, is the set of all expressions of the form

$$
\mathbf{a}_{0}+\mathbf{a}_{1} x+\cdots+\mathbf{a}_{m} x^{m} .
$$

Each element of $\mathcal{R}[X]$ is call a sequence polynomial. If $\mathbf{a}_{m}$ is a nonzero sequence, then $m$ is called the degree of $f$. If $\mathbf{a}_{i}=\left\{a_{i}\right\}_{n \geq 0}$ are constant sequences, then the sequence polynomial is called to be a constant sequence polynomial, and denoted briefly by $a_{0}+a_{1} x+\cdots+a_{n} x^{n}$.

A sequence polynomial can be considered as a map from $\mathcal{R}$ to $\mathcal{R}$. Let $\mathbf{u}$ be an integer sequence and $f(x)=\mathbf{a}_{0}+\mathbf{a}_{1} x+\cdots+\mathbf{a}_{m} x^{m}$ be a sequence polynomial with degree $m$ in $\mathcal{R}[X]$. Then the image of $\mathbf{u}$ 
under the map $f(x)$ is the integer sequence $f(\mathbf{u})=\mathbf{a}_{0}+\mathbf{a}_{1} \mathbf{u}+\cdots+\mathbf{a}_{m} \mathbf{u}^{m}$. In particular, if $f(x)=a_{0}+a_{1} x$, then $f(\mathbf{u})$ is called to be a linear polynomial of $\mathbf{u}$.

Definition 1. Given an integer sequence $\mathbf{u}=\{u(n)\}_{n \geq 0}$. If there exists an integer $k \geq 1$ and sequence polynomials $f_{i}(x) \in \mathcal{R}[X](0 \leq i<k)$ such that

$$
\{u(k n+i)\}_{n \geq 0}=f_{i}(\mathbf{u}),
$$

then we say that $\mathbf{u}$ is a polynomial generated sequence. The set of polynomials $\left\{f_{i}(x) \in \mathcal{R}[X]: 0 \leq\right.$ $i<k\}$ is called to be a generated polynomial system. The set of the sequences generated by polynomials $\left\{f_{i}(x) \in \mathcal{R}[X]: 0 \leq i<k\right\}$ is denoted by $\mathcal{G}\left(f_{0}, f_{1}, \cdots, f_{k-1}\right)$.

Assume $f_{i}(x)=\mathbf{a}_{i} x+\mathbf{b}_{i}$, where $\mathbf{a}_{i}=\left\{a_{i}(n)\right\}_{n \geq 0}, \mathbf{b}_{i}=\left\{b_{i}(n)\right\}_{n \geq 0}$ for $0 \leq i<k$. If $\mathbf{u}=\{u(n)\}_{n \geq 0} \in$ $\mathcal{G}\left(f_{0}, f_{1}, \cdots, f_{k-1}\right)$, then, for $0 \leq i<k, n \geq 0$,

$$
u(k n+i)=a_{i}(n) u(n)+b_{i}(n) .
$$

Hence, we have

$$
\begin{aligned}
u(0) & =\frac{b_{0}(0)}{1-a_{0}(0)}, \\
u(i) & =a_{i}(0) u(0)+b_{i}(0) \text { for } 1 \leq i<k, \\
\ldots & \ldots \ldots \ldots
\end{aligned}
$$

Note from above that $u(n)$ are determined by $a_{i}(n)$ and $b_{i}(n)$ for $0 \leq i<k, n \geq 0$. Moreover, if $u(0)$ is integer, then $\mathbf{u}$ is an integer sequence.

Hence, if the sequence polynomials $f_{i}(x) \in \mathcal{R}[X](0 \leq i<k)$ are degree 1 , we always assume $b_{0}(0)=$ $N\left(1-a_{0}(0)\right)$ for some integer $N$, and we define $u(0)=0$ if $a_{0}(0)=1$ in this paper. In this case, the set $\mathcal{G}\left(f_{0}, f_{1}, \cdots, f_{k-1}\right)$ always exists and has exactly one sequence $\mathbf{u}=\{u(n)\}_{n \geq 0}$, and we denote the polynomial generated sequence $\mathbf{u}$ by $\mathcal{G}\left(f_{0}, f_{1}, \cdots, f_{k-1}\right)$ briefly.

Example 1. (1) The polynomial generated sequence $\mathcal{G}(x,-x+1)=\{t(n)\}_{n \geq 0}$ is the famous ThueMorse sequence.

(2) Assume $k \geq 2$. The polynomial generated sequence

$$
\mathcal{G}(x, x+1 \cdots, x+k-1)=\left\{s_{k}(n)\right\}_{n \geq 0}
$$

is a $k$-regular sequence, where $s_{k}(n)$ is the sum of the digits in the base- $k$ representation of $n$.

So, given a generated polynomial system $\left\{f_{i}(x) \in \mathcal{R}[X]: 0 \leq i<k\right\}$, where the polynomials are degree 1 , we can obtain a set of integer sequences $\mathcal{G}\left(f_{0}, f_{1}, \cdots, f_{k-1}\right)$. Then, a problem appears. What can be said about properties of $\mathcal{G}\left(f_{0}, f_{1}, \cdots, f_{k-1}\right)$ ?

If $f_{i}(x)$ are linear polynomials for $0 \leq i<k$ with $k \geq 2$, then it is easy to check that $\mathcal{G}\left(f_{0}, f_{1}, \cdots, f_{k-1}\right)$ is a $k$-regular sequence. Moreover, if $f_{i}(x)=a_{i} x+\mathbf{b}_{i}$ for $0 \leq i<k$, where $a_{i}$ are integers and $\mathbf{b}_{i}$ are $k$-regular, then $\mathcal{G}\left(f_{0}, f_{1}, \cdots, f_{k-1}\right)$ is also $k$-regular. The following theorem gives a general result.

Theorem 1. Assume $k \geq 2$ and $f_{i}(x)=\mathbf{a}_{i} x+\mathbf{b}_{i}$ for $0 \leq i<k$. If $\mathbf{a}_{i}$ are $k$-automatic and $\mathbf{b}_{i}$ are $k$-regular, then the polynomial generated sequence $\mathcal{G}\left(f_{0}, f_{1}, \cdots, f_{k-1}\right)$ is $k$-regular.

Let $\mathbf{u}=\{u(n)\}_{n \geq 0}$ be a sequence, then we define the shift map $S(\mathbf{u})$ to be the sequence $\{u(n+1)\}_{n \geq 0}$. Similarly, we have $S^{k}(\mathbf{u})=u(k) u(k+1) u(k+2) \cdots$ for $k \geq 0$. Let $f=\mathbf{a}_{0}+\mathbf{a}_{1} x+\cdots+\mathbf{a}_{m} x^{m}$ be a sequence polynomial. Then, we define the composition $f \circ S(\mathbf{u})$, to be the sequence $f(S(\mathbf{u}))$.

Definition 2. Given an integer sequence $\mathbf{u}=\{u(n)\}_{n \geq 0}$. If there exist integers $k \geq 1, N \geq 0$, polynomials $f_{i}(x) \in \mathcal{R}[X]$ and shifts $S_{i} \in\left\{S^{j}: j \geq 0\right\}$ for $0 \leq i<\bar{k}$ such that

$$
u(k n+i)=f_{i} \circ S_{i}(\mathbf{u})(n),(n \geq N)
$$

then we say that $\mathbf{u}$ is a polynomial generated sequence with shift.

The following theorem tells us that the shift do not change the regularity of the polynomial generated sequence.

Theorem 2. Assume $k \geq 2$ and $f_{i}(x)=\mathbf{a}_{i} x+\mathbf{b}_{i}$ for $0 \leq i<k$. If $\mathbf{a}_{i}$ are $k$-automatic and $\mathbf{b}_{i}$ are $k$-regular, then the polynomial generated sequences with shift are $k$-regular.

In particular, we have 
Corollary 1. Let $\left\{a_{i}(n)\right\}_{n \geq 0}$ and $\left\{b_{i}(n)\right\}_{n \geq 0}$ be 2-automatic sequences for $0 \leq i<2$. Assume $\mathbf{u}=$ $\{u(n)\}_{n \geq 0}$ is a sequence defined by

$$
u(2 n)=a_{0}(n) u(n)+b_{0}(n), u(2 n+1)=a_{1}(n) u(n+1)+b_{1}(n) .
$$

Then the sequence $\mathbf{u}$ is 2-regular.

1.3. Hankel determinants of the characteristic sequence of the power of 2. Let $\mathbf{u}=\{u(n)\}_{n \geq 0}$ be a sequence of real numbers. For every integer $k \geq 0$, define a Hankel matrix $\mathbf{u}_{m, n}^{k}$ of order $m \times n$ associated with $\mathbf{u}$ as follows:

$$
\mathbf{u}_{m, n}^{k}=\left(\begin{array}{cccc}
u(k) & u(k+1) & \cdots & u(k+n-1) \\
u(k+1) & u(k+2) & \cdots & u(k+n) \\
\vdots & \vdots & \ddots & \vdots \\
u(k+m-1) & u(k+m) & \cdots & u(k+m+n-2)
\end{array}\right) .
$$

Note that the rows of $\mathbf{u}_{m, n}^{k}$ are made up of successive length- $n$ "windows" into the sequence $\mathbf{u}$. If $m=n$, we always use the symbols $\mathbf{u}_{n}^{k}$ and $\left|\mathbf{u}_{n}^{k}\right|$ to stand for the $n$-order Hankel matrix and $n$-order Hankel determinant respectively.

Hankel determinants associated with a sequence play an important role in the study of the moment problem, Padé approximation, and the combinatorial properties of sequence [5, 6, 14, 15, 16, Given a $k$-automatic integer sequence $\mathbf{u}=\{u(n)\}_{n \geq 0}$, we obtain a sequence of Hankel determinants $\left|\mathbf{u}_{n}^{m}\right|$. Note that the Hankel determinants $\left|\mathbf{u}_{n}^{m}\right|$ are determined by the block $u(m) u(m+1) \cdots u(m+2 n)$ for any fixed $n \geq 1$. And the block sequence $\{u(m) u(m+1) \cdots u(m+2 n)\}_{m \geq 0}$ is $k$-automatic. Hence, the determinant sequence $\left\{\left|\mathbf{u}_{n}^{m}\right|\right\}_{m \geq 0}$ is $k$-automatic, please see 4 .

There are some results about the automaticity of the Hankel determinant sequences. Allouche, Peyrière, Wen and Wen first studied the Hankel determinant of the Thue-Morse sequence $\mathbf{t}$ in [4. They proved that the sequences $\left\{\left|\mathbf{t}_{n}^{m}\right|(\bmod 2)\right\}_{n \geq 0}$ are 2-automatic. In the same way, Wen, Wu [19] and Guo, Wen 13 respectively studied the the Hankel determinants of the Cantor sequence $\mathbf{c}$ and the differences of Thue-Morse $\Delta^{k}(\mathbf{t})$. They proved that the sequences $\left\{\left|\mathbf{c}_{n}^{m}\right|(\bmod 3)\right\}_{n \geq 0}$ are 3 -automatic, and the the sequences $\left\{\left|\boldsymbol{\Delta}^{\mathbf{k}}(\mathbf{t})_{n}^{m}\right|(\bmod 2)\right\}_{n \geq 0}$ are 2-automatic.

Here, we point out that if $\{u(n)\}_{n \geq 0}$ is $k$-regular over $\mathbb{Z}$, then $\{u(n)(\bmod m)\}_{n \geq 0}$ is $k$-automatic for any $m \geq 1$. But the converse does not hold. For example, the sequence $\left\{2^{n}(\bmod m)\right\}_{n \geq 0}$ is $k$-automatic for any $m \geq 1, k \geq 2$, but the sequence $\left\{2^{n}\right\}_{n \geq 0}$ is not $k$-regular for any $k \geq 2$, please see [1]. Hence, although there are many sequences are either $k$-automatic or $k$-regular in [11], it is often quite challenging to determine the automaticity of the Hankel determinant sequences $\left\{\left|\mathbf{u}_{n}^{m}\right|\right\}_{n \geq 0}$.

In this paper, we consider the Hankel determinants of the characteristic sequence of powers 2

$$
\mathbf{p}=\{p(n)\}_{n \geq 0}=0110100010000 \cdots,
$$

where $p(n)=1$ if $n=2^{k}$ for some $k \geq 0$ and $p(n)=0$ otherwise.

Let $d(m, n)$ denote the $\left|\mathbf{p}_{n}^{m}\right|$ for $m \geq 0, n \geq 1$. Using polynomial generated sequnces, we prove that

Theorem 3. The sequence $\{d(0, n)\}_{n \geq 0}$ is 2-regular; The sequences $\{d(2 k, n)\}_{n \geq 0}$ are 2-automatic for all $k \geq 1$; The sequences $\{d(2 k+1, n)\}_{n \geq 0}$ are periodic for all $k \geq 0$.

The sequence $\mathbf{p}$ is a 2-automatic sequence, more about the sequence $\mathbf{p}$, please see [7]. Let $C_{n}=\frac{1}{n+1}\left(\begin{array}{c}2 n \\ n\end{array}\right)$ be a Catalan number, then $C_{n}(\bmod 2)=p(n+1)$ for all $n \geq 0$. Recently, using permutation, Cigler [8] studied the Hankel determinants of the sequence $\left\{C_{n}(\bmod 2)\right\}_{n \geq 0}=\{p(n+1)\}_{n \geq 0}$. By computer experiments, they have a conjecture which have been proved by us. We state it as follows.

Theorem 4 (Cigler [8]). Assume $m$ is an integer with $2^{k}<m \leq 2^{k+1}$ for some $k \geq 1$.

(1) If $m=2 r+1$, then $d\left(m, 2^{k+1} n\right)=1$ and $d\left(m, 2^{k+1} n-m+1\right)=(-1)^{r}$.

(2) If $m=2 r$, then $d\left(m, 2^{k+1} n\right)=d\left(2,2^{k+1} n\right)$ and $d\left(m, 2^{k+1} n-m+1\right)=(-1)^{n+\epsilon_{r}} d\left(2,2^{k+1} n-m+1\right)$, where $\left\{\epsilon_{r}\right\}_{r \geq 1}$ is a sequence over $\{0,1\}$ defined by

$$
\epsilon_{1}=1, \epsilon_{2 r}=\left(\epsilon_{r}+r\right) \bmod 2, \epsilon_{2 r+1}=\epsilon_{r+1},(r \geq 1) .
$$

The paper is organized as follows. In Section 2, we recall some notation briefly. In Section 3, we give a proof of Theorem 1 and Theorem 2 In the lase section, we study the Hankel determinants $d(m, n)$ and answer this conjecture by a precise recurrence formula of the determinants $d(m, n)$ for $m \geq 0, n \geq 1$. At last, we give a proof of Theorem 3 


\section{Preliminaries}

In this section, we briefly recall some notation and theorems. More notation, please see 3 .

In this paper, we always denote the set of non-negative integers by $\mathbb{N}$ and denote the set of integers by $\mathbb{Z}$. For any two set $A, B$ and number $c$, define $A+B=\{a+b: a \in A, b \in B\}, A B=\{a b: a \in A, b \in B\}$ and $c A=\{c a: a \in A\}$. We define $A^{0}=1$ and $A^{i}=A A^{i-1}, A^{-i}=\emptyset$ for any $i \geq 1$. Assume $f$ is map defined on $A$, then the image of $A$ under $f$ is denoted by $f(A)$, i.e., $f(A)=\{f(a): a \in A\}$. In particular, if $f(A) \subset A$, then we say that the set $A$ is invariant under the map $f$.

We always call a finite set $\Sigma$ alphabet and its elements letters. A word is made up of letters by the operation of concatenation. We denote the set of all finite words by $\Sigma^{*}$, including empty word $\epsilon$. Together with the operation of concatenation, $\Sigma^{*}$ forms a free monoid. An infinite sequence, denote by $\mathbf{u}=\{u(n)\}_{n \geq 0}=u(0) u(1) u(2) \cdots$, is a map from $\mathbb{N}$ to $\Sigma$. The set of all infinite sequences over $\Sigma$ is denoted by $\Sigma^{\mathbb{N}}$.

Among the infinite sequences, $k$-automatic and $k$-regular sequences satisfy a variety of useful properties. We recall some results from [1, 2].

Theorem 5. (1, Theorem 2.3) A sequence is $k$-regular and takes on only finitely many values if and only if it is k-automatic.

Theorem 6. (1], Theorem 2.5) Let $\{u(n)\}_{n \geq 0}$ and $\{v(n)\}_{n \geq 0}$ be k-regular sequences. Then so are $\{u(n)+v(n)\}_{n \geq 0},\{u(n) v(n)\}_{n \geq 0}$ and $\{c u(n)\}_{n \geq 0}$ for any $c$.

Theorem 7. (1], Theorem 2.10) Let $\{u(n)\}_{n \geq 0}$ be a k-regular sequence with values in $\mathbb{C}$, the set of complex numbers. Then there exists a constant $c$ such that $u(n)=\mathcal{O}\left(n^{c}\right)$.

Theorem 8. (2], Theorem 6) Let $\{u(n)\}_{n \geq 0}$ be a sequence with values in a Noetherian ring R. Suppose there exist integers $k \geq 2, t, r, n_{0}$ such that each sequence $\left\{u\left(k^{t+1} n+e\right)\right\}_{n \geq n_{0}}$ for $0 \leq e<k^{t+1}$ is a linear combination of the sequences $\left\{u\left(k^{i} n+j\right)\right\}_{n \geq n_{0}}$ with $0 \leq i \leq t, 0 \leq j<k^{i}$ and the sequences $\{u(n+p)\}_{n \geq n_{0}}$ with $0 \leq p \leq r$. Then the sequence $\{u(n)\}_{n \geq 0}$ is k-regular.

\section{Polynomial Generated Sequence}

In this section, we study the polynomial generated sequences and give a proof of Theorem [1] and Theorem 2 .

Let $f_{i}(x)=\mathbf{a}_{i} x+\mathbf{b}_{i}$ be sequence polynomials, where $\mathbf{a}_{i}=\left\{a_{i}(n)\right\}_{n \geq 0}, \mathbf{b}_{i}=\left\{b_{i}(n)\right\}_{n \geq 0}$ for $0 \leq i<k$. Let

$$
\mathcal{A}=\mathcal{K}_{k}\left(\mathbf{a}_{0}\right) \cup \mathcal{K}_{k}\left(\mathbf{a}_{1}\right) \cup \cdots \cup \mathcal{K}_{k}\left(\mathbf{a}_{k-1}\right)
$$

and

$$
\mathcal{B}=\mathcal{K}_{k}\left(\mathbf{b}_{0}\right) \cup \mathcal{K}_{k}\left(\mathbf{b}_{1}\right) \cup \cdots \cup \mathcal{K}_{k}\left(\mathbf{b}_{k-1}\right) .
$$

To prove Theorem 1 we need the following lemma.

Lemma 1. Let $\mathcal{S}$ be a finite set of integer sequences which take finitely many values, then the set $\bigcup_{i>0} \mathcal{S}^{i}$ is finitely generated. Moreover, if the sequences of $\mathcal{S}$ take values in $\{-1,0,1\}$, then $\bigcup_{i \geq 0} \mathcal{S}^{i}$ is a finite set.

Proof. Let $\mathbf{u}$ be an integer sequence over the alphabet $\left\{a_{0}, a_{1}, \cdots, a_{M}\right\}$, we define the characteristic sequences $\chi_{\mathbf{u}}^{j}$ for $0 \leq j \leq M$, by

$$
\chi_{\mathbf{u}}^{j}(n)= \begin{cases}1 & \text { if } u(n)=a_{j} \\ 0 & \text { otherwise. }\end{cases}
$$

Then $\mathbf{u}$ is a $\mathbb{Z}$-linear combination of a finite set $\left\{\chi_{\mathbf{u}}^{0}, \chi_{\mathbf{u}}^{1}, \cdots, \chi_{\mathbf{u}}^{M}\right\}$, i.e.,

$$
\mathbf{u}=a_{0} \chi_{\mathbf{u}}^{0}+a_{1} \chi_{\mathbf{u}}^{1}+\cdots+a_{M} \chi_{\mathbf{u}}^{M} .
$$

Note that $\chi_{\mathbf{u}}^{j}$ are binary sequences, so we have $\left(\chi_{\mathbf{u}}^{j}\right)^{k}=\chi_{\mathbf{u}}^{j}$ for all $k \geq 1$. Hence, $\mathbf{u}^{k}$ is a $\mathbb{Z}$-linear combination of at most $2^{M+1}$ sequences which are of the form

$$
\left(\chi_{\mathbf{u}}^{0}\right)^{i_{0}}\left(\chi_{\mathbf{u}}^{1}\right)^{i_{1}} \cdots\left(\chi_{\mathbf{u}}^{M}\right)^{i_{M}},
$$

where $i_{j} \in\{0,1\}$ for $0 \leq j \leq M$.

Now, assume $\mathcal{S}=\left\{\mathbf{u}_{0}, \mathbf{u}_{1}, \cdots, \mathbf{u}_{N}\right\}$. Then, for $i \geq 0$, we have

$$
\mathcal{S}^{i}=\left\{\mathbf{u}_{0}^{n_{0}} \mathbf{u}_{1}^{n_{1}} \cdots \mathbf{u}_{N}^{n_{N}}: n_{j} \geq 0 \text { for all } 0 \leq j \leq N \text { and } n_{0}+n_{1}+\cdots+n_{N}=i\right\} .
$$

So, each sequence $\mathbf{u}_{i}^{n_{i}}$ of $\mathcal{S}^{i}$ is a $\mathbb{Z}$-linear combination of a finite number of sequences. Hence, the set $\bigcup_{i \geq 0} \mathcal{S}^{i}$ is finitely generated. 
In particular, if a sequence $\mathbf{u}$ takes values in $\{-1,0,1\}$, then $\mathbf{u}^{k} \in\left\{\mathbf{u}, \mathbf{u}^{2}\right\}$ for all $k \geq 1$. Hence, if the sequences of $\mathcal{S}$ take values in $\{-1,0,1\}$, then $\mathbf{u}_{i}^{k} \in\left\{\mathbf{u}_{i}, \mathbf{u}_{i}^{2}\right\}$ for all $k \geq 1$ for each $0 \leq i \leq N$. Thus, $\bigcup_{i \geq 0} \mathcal{S}^{i}$ is a finite set.

Proof of Theorem [1. Assume $\mathbf{u}=\mathcal{G}\left(f_{0}, f_{1}, \cdots, f_{k-1}\right)$. Then, we have $u(k n+i)=a_{i}(n) u(n)+b_{i}(n)$ for $0 \leq i<k, n \geq 0$. Hence,

$$
\mathcal{K}_{k}(\mathbf{u}) \subset \bigcup_{i \geq 0}\left(\mathcal{A}^{i} \mathbf{u}+\mathcal{B} \sum_{s=0}^{i-1} \mathcal{A}^{s}\right)
$$

Since the sequences $\mathbf{a}_{i}=\left\{a_{i}(n)\right\}_{n \geq 0}$ are $k$-automatic and $\mathbf{b}_{i}=\left\{b_{i}(n)\right\}_{n \geq 0}$ are $k$-regular for $0 \leq i<k$, we know that the set $\mathcal{A}$ is finite and the set $\mathcal{B}$ is finitely generated. Hence, by Lemma $1 \bigcup_{i \geq 0} \mathcal{A}^{i} \mathbf{u}$ and $\bigcup_{i>0} \mathcal{B}\left(\sum_{s=0}^{i-1} \mathcal{A}^{s}\right)$ are finitely generated by some finite set. Thus, the $k$-kernel $\mathcal{K}_{k}(\mathbf{u})$ is finitely generated, which implies that the polynomial generated sequence $\mathcal{G}\left(f_{0}, f_{1}, \cdots, f_{k-1}\right)$ is $k$-regular.

Now, using Lemma 1, we prove Theorem 2,

Proof of Theorem 圆, Let $\mathbf{u}=\{u(n)\}_{n \geq 0}$ be a polynomial generated sequence with polynomials $f_{0}, f_{1}, \cdots, f_{k-1}$ and shifts $S_{i}$ for $0 \leq i<k$. Since $S_{i} \in\left\{S^{j}: j \geq 0\right\}$, we assume $S_{i}=S^{j_{i}}$, where $j_{i} \geq 0$ are integers for $0 \leq i<k$. By definition 2, for any $0 \leq i<k$ and $n \geq N$, we have

$$
u(k n+i)=a_{i}(n) u\left(n+j_{i}\right)+b_{i}(n) .
$$

Note that there are at most $k N$ terms of $\mathbf{u}$ which do not satisfy above formula (3.1). For $0 \leq i<k$, define a ultimately periodic sequence $\mathbf{c}_{i}=\left\{c_{i}(n)\right\}_{n \geq 0}$ by

$$
c_{i}(n)= \begin{cases}u(k n+i)-a_{i}(n) u\left(n+j_{i}\right)-b_{i}(n), & \text { if } 0 \leq n<N, \\ 0, & \text { otherwise. }\end{cases}
$$

Then, for all $n \geq 0$,

$$
u(k n+i)=a_{i}(n) u\left(n+j_{i}\right)+b_{i}(n)+c_{i}(n),(0 \leq i<k) .
$$

Let $r=\max \left\{j_{i}: 0 \leq i<k\right\}$ and $Q$ is a fixed number satisfying $Q \geq \frac{k(1+r)}{k-1}$. Define sets

$$
\begin{aligned}
\mathcal{U} & =\left\{\{u(n+t)\}_{n \geq 0}: 0 \leq t \leq Q\right\}, \\
\mathcal{A}_{Q} & =\bigcup_{s=0}^{k-1}\left\{\left\{a_{s}\left(k^{i}(n+t)+j\right)\right\}_{n \geq 0}: i \geq 0,0 \leq j<k^{i}, 0 \leq t \leq Q\right\}, \\
\mathcal{B}_{Q} & =\bigcup_{s=0}^{k-1}\left\{\left\{b_{s}\left(k^{i}(n+t)+j\right)\right\}_{n \geq 0}: i \geq 0,0 \leq j<k^{i}, 0 \leq t \leq Q\right\}, \\
\mathcal{C}_{Q} & =\bigcup_{s=0}^{k-1}\left\{\left\{c_{s}\left(k^{i}(n+t)+j\right)\right\}_{n \geq 0}: i \geq 0,0 \leq j<k^{i}, 0 \leq t \leq Q\right\} .
\end{aligned}
$$

We claim that,

$$
\mathcal{K}_{k}(\mathbf{u}) \subset \bigcup_{i \geq 0}\left(\mathcal{A}_{Q}^{i} \mathcal{U}+\left(\mathcal{B}_{\mathcal{Q}}+\mathcal{C}_{\mathcal{Q}}\right) \sum_{s=0}^{i-1} \mathcal{A}_{Q}^{s}\right)
$$

To prove this claim, we need some maps. For $0 \leq \ell<k$, define $\psi_{\ell}:\{w(n)\}_{n \geq 0} \rightarrow\{w(k n+\ell)\}_{n \geq 0}$. Then, we only need to show that $\psi_{\ell}(\mathcal{U}) \subset \mathcal{A}_{Q} \mathcal{U}+\bar{B}_{Q}+\mathcal{C}_{Q}$ and the sets $\mathcal{A}_{Q}, \mathcal{B}_{Q}$ and $\mathcal{C}_{Q}$ are invariant under the maps $\psi_{\ell}$.

For any $0 \leq t \leq Q$ and $0 \leq \ell<k$, assume $t+\ell=k x+y$, where $0 \leq y<k$. So, by the choice of $Q$ and note that $0 \leq j_{y} \leq r$, we have

$$
0 \leq x \leq x+j_{y} \leq \frac{t+\ell}{k}+r \leq \frac{k-1+Q}{k}+r \leq Q .
$$

Then, we have the following cases.

- $u(k n+\ell+t)=u(k(n+x)+y)=a_{y}(n+x) u\left(n+x+j_{y}\right)+b_{y}(n+x)+c_{y}(n+x)$, so we have $\psi_{\ell}(\mathcal{U}) \subset \mathcal{A}_{Q} \mathcal{U}+\mathcal{B}_{Q}+\mathcal{C}_{Q}$

- For any $i \geq 0,0 \leq j<k^{i}$, we have $a_{s}\left(k^{i}(k n+\ell+t)+j\right)=a_{s}\left(k^{i}(k(n+x)+y)+j\right)=$ $a_{s}\left(k^{i+1}(n+x)+k^{i} y+j\right)$. Note that $0 \leq k^{i} y+j<k^{i+1}$, we have $\psi_{\ell}\left(\mathcal{A}_{Q}\right) \subset \mathcal{A}_{Q}$. In the same way, $\psi_{\ell}\left(\mathcal{B}_{Q}\right) \subset \mathcal{B}_{Q}$ and $\psi_{\ell}\left(\mathcal{B}_{Q}\right) \subset \mathcal{B}_{Q}$. 
Hence, our claim holds.

If the sequences $\mathbf{a}_{i}=\left\{a_{i}(n)\right\}_{n \geq 0}$ are $k$-automatic and $\mathbf{b}_{i}=\left\{b_{i}(n)\right\}_{n \geq 0}$ are $k$-regular for $0 \leq i<k$, then the set $\mathcal{A}_{Q}$ is finite and the set $\mathcal{B}_{Q}$ is finitely generated. Note that the sets $\mathcal{U}$ and $\mathcal{C}_{Q}$ are finite, by Lemma 1, there exists a finite set $\mathcal{S}$ such that the sets

$$
\bigcup_{i \geq 0}\left(\mathcal{A}_{Q}^{i} \mathcal{U}+\left(\mathcal{B}_{\mathcal{Q}}+\mathcal{C}_{\mathcal{Q}}\right) \sum_{s=0}^{i-1} \mathcal{A}_{Q}^{s}\right)
$$

are $\mathbb{Z}$-linear combination of $\mathcal{S}$. Hence, the $k$-kernel $\mathcal{K}_{k}(\mathbf{u})$ is finitely generated and our theorem follows.

Remark 1. Note from the proof of Theorem 2 that the condition of Definition 1 can be weakened by $u(k n+i)=f_{i}(\mathbf{u})(n)(n \geq N)$ for some integer $N \geq 0$. Denote

$$
\mathcal{G}_{N}\left(f_{0}, f_{1}, \cdots, f_{k-1}\right)=\left\{\{u(n)\}_{n \geq 0}: u(k n+i)=f_{i}(\mathbf{u})(n), n \geq N \text { for } 0 \leq i \leq k\right\} .
$$

If $f_{i}=\mathbf{a}_{i} x+\mathbf{b}_{i}$, where $\mathbf{a}_{i}$ are $k$-automatic and $\mathbf{b}_{i}$ are $k$-regular for $0 \leq i \leq k$, then for every $N \geq 0$, the sequences of $\mathcal{G}_{N}\left(f_{0}, f_{1}, \cdots, f_{k-1}\right)$ are $k$-regular.

We end this section by some examples. Example 2 implies that the condition that all polynomials are degree 1 in Theorem [1, is necessary. Example 3] shows that if we replace the $k$-automatic condition of the sequence $\mathbf{a}_{i}$ by a $k$-regular condition in Theorem 1 then the polynomial generated sequence maybe not $k$-regular.

Example 2. The sequences in $\mathcal{G}\left(x^{2}, x+1\right)$ are not 2 -regular. Assume $\{u(n)\}_{n \geq 0} \in \mathcal{G}\left(x^{2}, x+1\right)$, and

$$
u(2 n)=u(n)^{2}, u(2 n+1)=u(n)+1 .
$$

If $u(0)=0$, then $u(3)=2$. If $u(0)=1$, then $u(2)=2$. In either case, $a \in\{2,3\}$, we have

$$
\frac{\log _{2}\left(u\left(a \cdot 2^{k}\right)\right)}{\log _{2}\left(a \cdot 2^{k}\right)}=\frac{2^{k} \log _{2}(u(a))}{k\left(\log _{2} a+1\right)}=\frac{2^{k}}{\left(1+\log _{2} a\right) k} \rightarrow \infty, \quad(k \rightarrow \infty) .
$$

Hence, by Theorem 7 , the sequences in $\mathcal{G}\left(x^{2}, x+1\right)$ are not 2 -regular.

Example 3. The sequence $\mathcal{G}(\mathbf{v} x, x+1)$ is not 2-regular, where $\mathbf{v}=\{n\}_{n \geq 0}$ is a 2-regular sequence. Assume $\{u(n)\}_{n \geq 0}=\mathcal{G}(\mathbf{v} x, x+1)$, then

$$
u(2 n)=n u(n), u(2 n+1)=u(n)+1 .
$$

It is easy to check that $u\left(2^{k} n\right)=2^{k-1+k-2+\cdots+1} n^{k} u(n)=2^{k(k-1) / 2} n^{k} u(n)$. Since $u(3)=2$ and

$$
\frac{\log _{2}\left(u\left(3 \cdot 2^{k}\right)\right)}{\log _{2}\left(3 \cdot 2^{k}\right)}=\frac{k(k-1) / 2+k \log _{2} 3+1}{k\left(\log _{2} 3+1\right)} \rightarrow \infty,(k \rightarrow \infty)
$$

which implies the sequence $\mathcal{G}(\mathbf{v} x, x+1)$ is not 2 -regular, by Theorem 7 ,

Example 4. Let $\mathbf{t}=\{t(n)\}_{n \geq 0}=01101001 \cdots$ be the Thue-Morse sequence. Clearly that $t(2 n)=$ $t(n), t(2 n+1)=1-t(n)$ for all $n \geq 0$. Let $\mathbf{u}=\{u(n)\}_{n \geq 0}=\mathcal{G}(\mathbf{t} x+1, x+\mathbf{t})$. Then, for $n \geq 0$,

$$
\begin{aligned}
& u(0)=u(1)=1, \\
& u(4 n)=u(4 n+1)=u(2 n)+u(2 n+1)-u(n), \\
& u(4 n+2)=-u(2 n)+u(n)+2, \\
& u(4 n+3)=u(n)+1 .
\end{aligned}
$$

Hence, by Theorem $8, \mathcal{G}(\mathbf{t} x+1, x+\mathbf{t})$ is a 2-regular sequence.

\section{Application}

In this section, we study the Hankel determinants $d(m, n)$ and obtain the recurrence formulae of the determinants $d(m, n)$ for $m \geq 0, n \geq 1$. By these formulae, we prove the conjecture of Cigler. Moreover, we find that the Hankel determinant sequence $\{d(0, n)\}_{n \geq 0}$ is a polynomial generated sequence with shift. Using Corollary 1, we give a proof of Theorem 3 at last.

Let $\mathbf{p}=\{p(n)\}_{n \geq 0}$ be the characteristic sequence of powers 2. Recall that the sequence $\{p(n)\}_{n \geq 0}$ can be generated by the recurrence formula:

$$
p(0)=0, p(1)=1, p(2 n)=p(n), p(2 n+1)=0,(n \geq 1) .
$$

Let $d(m, n)=\left|\mathbf{p}_{n}^{m}\right|=\operatorname{det}(p(i+j+m))_{i, j=0}^{n-1}$ for any $m \geq 0, n \geq 1$, then we have the following lemma which plays important role in this paper.

Lemma 2. For any $m \geq 1, n \geq 1$, we have 
(1) $d(0,2 n)=d(0, n) d(1, n)-d(2, n-1) d(3, n-1)$,

(2) $d(0,2 n+1)=d(0, n+1) d(1, n)-d(2, n) d(3, n-1)$,

(3) $d(1,2 n)=(-1)^{n} d^{2}(1, n)$,

(4) $d(1,2 n+1)=(-1)^{n} d^{2}(2, n)$,

(5) $d(2 m, 2 n)=d(m, n) d(m+1, n)$,

(6) $d(2 m, 2 n+1)=d(m, n+1) d(m+1, n)$,

(7) $d(2 m+1,2 n)=(-1)^{n} d^{2}(m+1, n)$,

(8) $d(2 m+1,2 n+1)=0$.

Here, we define $d(2,0)=d(3,0)=1$.

Proof. For each $n$-order square matrix $M=\left(m_{i, j}\right)_{1 \leq i, j \leq n}$, there exists a matrix $U$ with $|U|= \pm 1$ such that

$$
U M U^{t}=\left(\begin{array}{cc}
\left(m_{2 i-1,2 j-1}\right)_{\substack{1 \leq i \leq \mu \\
1 \leq j \leq \mu}} & \left(m_{2 i-1,2 j}\right)_{\substack{1 \leq i \leq \mu \\
1 \leq j \leq \nu \\
\\
1 \leq i \leq \nu}} \\
\left(m_{2 i, 2 j-1}\right)_{1 \leq j \leq \nu} & \left(m_{2 i, 2 j}\right)_{\substack{1 \leq i \leq \nu \\
1 \leq j \leq \nu}}
\end{array}\right),
$$

where $\mu=\left\lfloor\frac{1}{2}(n+1)\right\rfloor$ and $\nu=\left\lfloor\frac{1}{2} n\right\rfloor$ and $U^{t}$ denote the transposed matrix of $U$.

(1) By Formula (4.1) and (4.2), we have

$$
U \mathbf{p}_{2 n}^{0} U^{t}=\left(\begin{array}{cc}
\mathbf{p}_{n}^{0} & A_{n, n} \\
A_{n, n} & \mathbf{p}_{n}^{1}
\end{array}\right)
$$

where $A_{m, n}=\left(a_{i j}\right)_{1 \leq i \leq m, 1 \leq j \leq n}$ denote the $m \times n$ matrix with all entries are zero except $a_{11}=1$. Hence,

$$
\left|\mathbf{p}_{2 n}^{0}\right|=\left|\begin{array}{cc}
\mathbf{p}_{n}^{0} & A_{n, n} \\
A_{n, n} & \mathbf{p}_{n}^{1}
\end{array}\right|=\left|\mathbf{p}_{n}^{0}\right|\left|\mathbf{p}_{n}^{1}\right|-\left|\mathbf{p}_{n-1}^{2}\right|\left|\mathbf{p}_{n-1}^{3}\right| .
$$

(2) By Formula (4.1) and (4.2), we have

$$
\left|\mathbf{p}_{2 n+1}^{0}\right|=\left|\begin{array}{cc}
\mathbf{p}_{n+1}^{0} & A_{n+1, n} \\
A_{n, n+1} & \mathbf{p}_{n}^{1}
\end{array}\right|=\left|\mathbf{p}_{n+1}^{0}\right|\left|\mathbf{p}_{n}^{1}\right|-\left|\mathbf{p}_{n}^{2}\right|\left|\mathbf{p}_{n-1}^{3}\right| .
$$

(3) By Formula (4.1) and (4.2), we have

$$
\left|\mathbf{p}_{2 n}^{1}\right|=\left|\begin{array}{cc}
A_{n, n} & \mathbf{p}_{n}^{1} \\
\mathbf{p}_{n}^{1} & \mathbf{0}_{n, n}
\end{array}\right|=(-1)^{n}\left|\mathbf{p}_{n}^{1}\right|^{2} .
$$

where $\mathbf{0}_{m, n}$ denote the $m \times n$ matrix with all entries are zero.

(4) By Formula (4.1) and (4.2), we have

$$
\left|\mathbf{p}_{2 n+1}^{1}\right|=\left|\begin{array}{cc}
A_{n+1, n+1} & \mathbf{p}_{n+1, n}^{1} \\
\mathbf{p}_{n, n+1}^{1} & \mathbf{0}_{n, n}
\end{array}\right|=(-1)^{n}\left|\mathbf{p}_{n}^{2}\right|^{2} .
$$

(5) By Formula (4.1) and (4.2), we have

$$
\left|\mathbf{p}_{2 n}^{2 m}\right|=\left|\begin{array}{cc}
\mathbf{p}_{n}^{m} & \mathbf{0}_{n, n} \\
\mathbf{0}_{n, n} & \mathbf{p}_{n}^{m+1}
\end{array}\right|=\left|\mathbf{p}_{n}^{m}\right|\left|\mathbf{p}_{n}^{m+1}\right| .
$$

(6) By Formula (4.1) and (4.2), we have

$$
\left|\mathbf{p}_{2 n}^{2 m+1}\right|=\left|\begin{array}{cc}
\mathbf{0}_{n, n} & \mathbf{p}_{n}^{m+1} \\
\mathbf{p}_{n}^{m+1} & \mathbf{0}_{n, n}
\end{array}\right|=(-1)^{n}\left|\mathbf{p}_{n}^{m+1}\right|^{2}
$$

(7) By Formula (4.1) and (4.2), we have

$$
\left|\mathbf{p}_{2 n+1}^{2 m}\right|=\left|\begin{array}{cc}
\mathbf{p}_{n+1}^{m} & \mathbf{0}_{n+1, n} \\
\mathbf{0}_{n, n+1} & \mathbf{p}_{n}^{m+1}
\end{array}\right|=\left|\mathbf{p}_{n+1}^{m}\right|\left|\mathbf{p}_{n}^{m+1}\right| .
$$

(8) By Formula (4.1) and (4.2), we have

$$
\left|\mathbf{p}_{2 n+1}^{2 m+1}\right|=\left|\begin{array}{cc}
\mathbf{0}_{n+1, n+1} & \mathbf{p}_{n+1, n}^{m+1} \\
\mathbf{p}_{n, n+1}^{m+1} & \mathbf{0}_{n, n}
\end{array}\right|=0
$$

Remark 2. Define $d(m, 0)=1, d(m,-1)=0$ for all $m \geq 0$, then Formulae of Lemma Q hold for $n \geq 0$.

Proposition 1. For any $n \geq 1, d(1, n), d(2, n) \in\{-1,1\}, d(m, n) \in\{-1,0,1\}(m \geq 3)$. Moreover, $d(1, n)=(-1)^{\left\lfloor\frac{n}{2}\right\rfloor}$. 
Proof. We first prove that $d(1, n), d(2, n) \in\{-1,1\}$ by induction on $n$. It is easy to check that $d(1,1)=$ $-d(1,2)=-d(1,3)=1, d(2,1)=d(2,2)=-d(2,3)=1$. Now, assume that $d(1, n), d(2, n) \in\{-1,1\}$ for all $n<2^{k}$ with $k \geq 1$. Then, for any $2^{k} \leq n<2^{k+1}$, there exists an integer $m<2^{k}$ such that $n=2 m$ or $n=2 m+1$. Moreover,

- $d(1, n)=d(1,2 m)=(-1)^{m} d^{2}(1, m) \in\{-1,1\}$,

- $d(1, n)=d(1,2 m+1)=(-1)^{m} d^{2}(2, m) \in\{-1,1\}$,

- $d(2, n)=d(1,2 m)=d(1, m) d(2, m) \in\{-1,1\}$,

- $d(2, n)=d(1,2 m+1)=d(1, m+1) d(2, m) \in\{-1,1\}$.

Hence, $d(1, n), d(2, n) \in\{-1,1\}$ for all $n \geq 1$.

Now, assume there exists an integer $k$ such that $d(m, n) \in\{-1,0,1\}$ for all $m \leq 2^{k}, n \geq 1$. We need to prove the conclusion hold for $m \leq 2^{k+1}$. Since $m=2 s$ or $m=2 s+1$ for some $s \leq 2^{k}$, by Lemma 2 and the hypothesis, we have

- $d(m, 2 n)=d(2 s, 2 n)=d(s, n) d(s+1, n) \in\{-1,0,1\}$,

- $d(m, 2 n+1)=d(2 s, 2 n+1)=d(s, n+1) d(s+1, n) \in\{-1,0,1\}$,

- $d(m, 2 n)=d(2 s+1,2 n)=(-1)^{n} d^{2}(s+1, n) \in\{-1,0,1\}$,

- $d(m, 2 n+1)=d(2 s+1,2 n+1)=0 \in\{-1,0,1\}$.

Thus, $d(m, n) \in\{-1,0,1\}$ for all $m, n \geq 1$.

By Lemma 2, it follows that $d(1, n)=(-1)^{\left\lfloor\frac{n}{2}\right\rfloor}$, which completes this proof.

The following two propositions have been proved by Cigler in [8]. Here, we give another proofs of them. Our method which is different from Cigler mainly depends on the recurrence formulae. The first proposition gives a description of the sequence $\{d(2, n)\}_{n \geq 0}$. The second proposition gives a description of the sequence $\{d(m, n)\}_{n \geq 0}$ for all $m \geq 3$.

Proposition 2. If $2^{k} \leq n<2^{k+1}$ for some integer $k \geq 2$, then

$$
d(2, n)=\left\{\begin{array}{l}
-d\left(2, n-2^{k}\right) \quad \text { if } 2^{k} \leq n<2^{k}+2^{k-1}, \\
d\left(2, n-2^{k}\right) \quad \text { if } 2^{k}+2^{k-1} \leq n<2^{k+1} .
\end{array}\right.
$$

Proof. For $k=2$, the assertions above can be checked directly. Assume the proposition is true for $k \leq N$. Now, we discuss the case $k=N+1$.

If $2^{N+1} \leq n<2^{N+1}+2^{N}$ and $n=2^{N+1}+2 m$, then $0 \leq m<2^{N-1}$. By Lemma 2 and the hypothesis, we have $d\left(2,2^{N}+m\right)=-d(2, m)$ and

$$
\begin{aligned}
d(2, n) & =d\left(2,2^{N+1}+2 m\right)=d\left(1,2^{N}+m\right) d\left(2,2^{N}+m\right) \\
& =-d\left(1,2^{N}+m\right) d(2, m)=-d(1, m) d(2, m) \\
& =-d(2,2 m)=-d\left(2, n-2^{N+1}\right) .
\end{aligned}
$$

The other ones can be obtained by the same method.

Remark 3. In fact, Proposition 2 gives a generation method of the sequence $\{d(2, n)\}_{n \geq 0}$. Let $A_{0}=$ $11, B_{0}=1-1, A_{n}=A_{n-1} B_{n-1}, B_{n}=\overline{A_{n-1}} B_{n-1}(n \geq 1)$, where the overbar is shorthand for the morphism that maps 1 to -1 and -1 to 1 . Then,

$$
\{d(2, n)\}_{n \geq 0}=\lim _{n \rightarrow \infty} A_{n}=A_{0} B_{0} \overline{A_{0}} B_{0} \overline{A_{0}} \overline{B_{0}} \overline{A_{0}} B_{0} \cdots=111-1-1-11-1-1-1-11 \cdots .
$$

Proposition 3. If $2^{k}<m \leq 2^{k+1}$ for some integer $k \geq 1$, then

$$
d(m, n)= \begin{cases} \pm 1, & \text { if } n \equiv 0 \text { or } 1-m\left(\bmod 2^{k+1}\right), \\ 0, & \text { otherwise. }\end{cases}
$$

Proof. By (7), (8) of Lemma 2 and Proposition 1] we have

$$
d(3,2 n)=(-1)^{n} d^{2}(2, n)=(-1)^{n}, d(3,2 n+1)=0 .
$$

Then, by (5), (6) of Lemma 2, we have

- $d(4,4 n)=d(2,2 n) d(3,2 n)=(-1)^{n} d(2,2 n)$,

- $d(4,4 n+1)=d(2,2 n+1) d(3,2 n)=(-1)^{n} d(2,2 n+1)$,

- $d(4,4 n+2)=d(2,2 n+1) d(3,2 n+1)=0$,

- $d(4,4 n+3)=d(2,2 n+2) d(3,2 n+1)=0$. 
Hence, $d(3, n) \neq 0 \Leftrightarrow n \equiv 0$ or $2(\bmod 4), d(4, n) \neq 0 \Leftrightarrow n \equiv 0$ or $1(\bmod 4)$, which implies that the conclusions hold for $k=1$.

Now, assume the assertions hold for $k \leq N$, we need to prove the case $k=N+1$. There are following cases to discuss.

- If $2^{N+1}<m<2^{N+2}$ and $m=2 r$ for some integer $r$, then $2^{N}<r, r+1 \leq 2^{N+1}$. By Lemma 2 and the hypothesis, we have

$-d(2 r, 2 s) \neq 0 \Leftrightarrow d(r, s) d(r+1, s) \neq 0 \Leftrightarrow s \equiv 0\left(\bmod 2^{N+1}\right) \Leftrightarrow 2 s \equiv 0\left(\bmod 2^{N+2}\right)$.

$-d(2 r, 2 s+1) \neq 0 \Leftrightarrow d(r, s+1) d(r+1, s) \neq 0 \Leftrightarrow s \equiv-r\left(\bmod 2^{N+1}\right) \Leftrightarrow 2 s+1 \equiv 1-$ $2 r\left(\bmod 2^{N+2}\right)$.

- If $m=2^{N+2}$ and $m=2 r$, then $r=2^{N+1}, 2^{N+1}<r+1 \leq 2^{N+2}$. We have $d(r+1, s) \neq 0 \Leftrightarrow s \equiv$ 0 or $2^{N+1}\left(\bmod 2^{N+2}\right) \Leftrightarrow s \equiv 0\left(\bmod 2^{N+1}\right)$. Hence, by Lemma 2 and the hypothesis, we have

$-d(2 r, 2 s) \neq 0 \Leftrightarrow d(r, s) d(r+1, s) \neq 0 \Leftrightarrow s \equiv 0\left(\bmod 2^{N+1}\right) \Leftrightarrow 2 s \equiv 0\left(\bmod 2^{N+2}\right)$.

$-d(2 r, 2 s+1) \neq 0 \Leftrightarrow d(r, s+1) d(r+1, s) \neq 0 \Leftrightarrow s \equiv 0\left(\bmod 2^{N+1}\right) \Leftrightarrow 2 s+1 \equiv 1 \equiv$ $1-2 r\left(\bmod 2^{N+2}\right)$.

- If $2^{N+1}<m \leq 2^{N+2}$ and $m=2 r+1$ for some integer $r$, then $2^{N}<r+1 \leq 2^{N+1}$. By Lemma 2 and the hypothesis, we have

$d(2 r+1,2 s) \neq 0 \Leftrightarrow d(r+1, s) \neq 0 \Leftrightarrow s \equiv 0$ or $-r\left(\bmod 2^{N+1}\right) \Leftrightarrow 2 s \equiv 0$ or $-2 r\left(\bmod 2^{N+2}\right)$.

Thus, if $2^{N+1}<m \leq 2^{N+2}$, then

$$
d(m, n) \neq 0 \Leftrightarrow n \equiv 0 \text { or } 1-m\left(\bmod 2^{N+2}\right),
$$

which completes the proof.

Now, we give a proof of Theorem 4 which is an answer of Cigler's conjecture.

Proof of Theorem 4 . It is easy to check that that the two assertions hold for $k=1$. Assume the two assertions hold for $k \leq N(N \geq 1)$, it suffices to show that the assertions also hold for $k=N+1$. There are three cases to discuss.

- If $2^{N+1}<m \leq 2^{N+2}$ and $m=2 r+1$, then, by (7) of Lemma 2, we have

$-d\left(m, 2^{N+2} n\right)=d\left(2 r+1,2^{N+2} n\right)=(-1)^{2^{N+1} n}=1$,

$-d\left(m, 2^{N+2} n-m+1\right)=d\left(2 r+1,2^{N+2} n-2 r\right)=(-1)^{2^{N+1} n-r}=(-1)^{r}$.

- If $2^{N+1}<m \leq 2^{N+2}$ and $m=4 r+2$, then $2^{N}<2 r+1,2 r+2 \leq 2^{N+1}$.

- Note that $d\left(2,2^{N+2} n\right)=d\left(2,2^{N+1} n\right)$. By (5) of Lemma 2 and the hypothesis, we have

$$
\begin{aligned}
d\left(m, 2^{N+2} n\right) & =d\left(4 r+2,2^{N+2} n\right)=d\left(2 r+1,2^{N+1} n\right) d\left(2 r+2,2^{N+2} n\right) \\
& =d\left(2,2^{N+1} n\right)=d\left(2,2^{N+2} n\right) .
\end{aligned}
$$

- Note that $d\left(2,2^{N+2} n-4 r-1\right)=(-1)^{r} d\left(2,2^{N+1} n-2 r-1\right)$ and $\epsilon_{2 r+1}=\epsilon_{r+1}$. By $(6)$ of Lemma 2 and the hypothesis, we have

$$
\begin{aligned}
d\left(m, 2^{N+2} n-m+1\right) & =d\left(4 r+2,2^{N+2} n-4 r-1\right) \\
& =d\left(2 r+1,2^{N+1} n-2 r\right) d\left(2 r+2,2^{N+1} n-2 r-1\right) \\
& =(-1)^{r}(-1)^{n+\epsilon_{r+1}} d\left(2,2^{N+1} n-2 r-1\right) \\
& =(-1)^{n+\epsilon_{2 r+1}} d\left(2,2^{N+2} n-4 r-1\right) .
\end{aligned}
$$

- If $2^{N+1}<m \leq 2^{N+2}$ and $m=4 r$, then $2^{N}<2 r \leq 2^{N+1}, 2^{N}+1<2 r+1 \leq 2^{N+1}+1$.

- Note that $d\left(2,2^{N+2} n\right)=d\left(2,2^{N+1} n\right)$. By (5) of Lemma 2, the first case and the hypothesis, we have

$$
\begin{aligned}
d\left(m, 2^{N+2} n\right) & =d\left(4 r, 2^{N+2} n\right)=d\left(2 r, 2^{N+1} n\right) d\left(2 r+1,2^{N+2} n\right) \\
& =d\left(2 r, 2^{N+1} n\right)=d\left(2,2^{N+1} n\right)=d\left(2,2^{N+2} n\right) .
\end{aligned}
$$

- Note that $d\left(2,2^{N+2} n-4 r+1\right)=(-1)^{r} d\left(2,2^{N+1} n-2 r\right)=d\left(2,2^{N+1} n-2 r+1\right)$ and $\epsilon_{2 r}=$ $\left(\epsilon_{r}+r\right) \bmod 2$. By $(6)$ of Lemma 2 and the hypothesis, we have

$$
\begin{aligned}
d\left(m, 2^{N+2} n-m+1\right) & =d\left(4 r, 2^{N+2} n-4 r+1\right) \\
& =d\left(2 r, 2^{N+1} n-2 r+1\right) d\left(2 r+1,2^{N+2} n-2 r\right) \\
& =(-1)^{r}(-1)^{n+\epsilon_{r}} d\left(2,2^{N+1} n-2 r+1\right) \\
& =(-1)^{n+\epsilon_{2 r}} d\left(2,2^{N+2} n-4 r+1\right) .
\end{aligned}
$$

Hence, our theorem follows.

By Lemma 2, Proposition 1, 3 and Theorem 4, we prove Therorem 3. 
Proof of Therorem 3. Let $\mathbf{a}=\{d(1, n)\}_{n \geq 0}, \mathbf{b}=\{d(2, n-1) d(3, n-1)\}_{n \geq 0}$ and $\mathbf{c}=\{d(2, n) d(3, n-$ 1) $\}_{n \geq 0}$. By Proposition 1 2 we know that $\{d(1, n)\}_{n \geq 0}$ is periodic with period 2 and $\{d(2, n)\}_{n \geq 0}$ is 2 -automatic. Hence, by Theorem [6, we know that $\mathbf{a}, \mathbf{b}$ and $\mathbf{c}$ are 2-automatic sequences. By Lemma 2. the sequence $\{d(0, n)\}_{n>0}$ is a polynomial generated sequence with shift, as

$$
d(0,2 n)=a(n) d(0, n)-b(n), d(0,2 n+1)=a(n) d(0, n+1)-c(n) .
$$

By Corollary 1, the sequene $\{d(0, n)\}_{n \geq 0}$ is 2-regular.

If $m \geq 3$ is odd, then by Proposition 3 and Theorem 4, we know that the sequence $\{d(m, n)\}_{n \geq 0}$ is periodic. Moreover, $2^{k+1}$ is a period if $2^{k}<m \leq 2^{k+1}$. If $m \geq 3$ is even, then by Proposition 3 and Theorem 4, we know that the sequence $\{d(m, n)\}_{n \geq 0}$ is 2 -automtic. We completes this proof.

Remark 4. Note from Proposition 1 that $d(m, n) \in\{-1,0,1\}$, we know that

$$
d^{k}(m, n) \in\left\{d(m, n), d^{2}(m, n)\right\}
$$

for any integer $k \geq 0$. By Lemma 2, it is easy to check directly that the 2-kernel of the sequence $\{d(m, n)\}_{m>1, n>0}$ is finite and the 2-kernel of the sequence $\{d(m, n)\}_{m>0, n>0}$ is finitely generated.

Hence, the two-dimensional sequence $\{d(m, n)\}_{m \geq 1, n \geq 0}$ is 2-automatic and $\{d(m, n)\}_{m \geq 0, n \geq 0}$ is 2 regular. An immediate consequence of a result of Salon in [17, 18, is that the sequences $\{\bar{d}(m, n)\}_{n>0}$ are 2-automatic for all $m \geq 1$. More about multidimensional automatic sequences and regular sequences, please see [3, 2].

\section{REFERENCES}

[1] J.-P. Allouche and J. Shallit, The ring of k-regular sequences, Theoret. Comput. Sci. 98 (1992), $163-197$.

[2] J.-P. Allouche and J. Shallit, The ring of k-regular sequences, II, Theoret. Comput. Sci. 307 (2003), 3-29.

[3] J.-P. Allouche and J. Shallit, Automatic Sequence. Theory, applications, generalizations, Cambridge University Press, Cambridge, 2003.

[4] J.-P. Allouche, J. Peyrière, Z.-X. Wen, and Z.-Y. Wen, Hankel determinants of the Thue-Morse sequence, Ann. Inst. Fourier (Grenoble). 48 (1998), 1-27.

[5] G.-A. Baker Jr., P. Graves-Morris, Padé Approximants, second edition, Encyclopedia of Mathematics and Its Applications, vol. 59, Cambridge University Press, Cambridge, 1996, xiv+746 pp.

[6] Y. Bugeaud, G.-N. Han, Z.-Y. Wen, J.-Y. Yao, Hankel determinants, Padé approximations and irrationality exponents, Int. Math. Res. Notes. V. 5 (2016), 1467-1496.

[7] V. Bruyère, G. Hansel, C. Michaux and R. Villemaire, Logic and p-recognizable sets of integers, Bull. Belgian Math. Soc. 1 (1994), 191-238. Corrigendum, Bull, Belgian Math. Soc. 1 (1994), 577.

[8] J. Cigler, A curious class of Hankel determinants, (2018), 32 pages, http://arxiv.org/abs/1803.05164

[9] G. Christol, T. Kamae, M. Mendès France and G. Rauzy, Suites algébriques, automates et substitutions, Bull. Soc. Math. France. 108 (4) (1980), 401-419.

[10] A. Cobham, Uniform tag sequences, Math. Systems Theory. 6 (1972), 164-192.

[11] É. Charlier, N. Rampersad, J. Shallit, Enumeration and decidable properties of automatic sequences, Internat. J. Found. Comput. Sci. 23 (05) (2012), 1035-1066.

[12] S. Eilenberg, Automata Languages and Machines Vol. A. Academic Press [A subsidiary of Harcourt Brace Jovanovich, Publishers], New York, 1974. Pure and Applied Mathematics, Vol. 58.

[13] Y.-J. Guo and Z.-X. Wen, Automaticity of the Hankel determinants of difference sequences of the Thue-Morse sequence, Theoret. Comput. Sci. 552 (2014), 1-12.

[14] Y.-J. Guo, Z.-X. Wen and W. Wu, On the irrationality exponent of the regular paperfolding numbers, Linear Algebra Appl. 446 (2014), 237-264.

[15] M. Lothaire, Algebraic combinatorics on words, Encyclopedia Math. Appl. 90, Cambridge Univ. Press, 2002.

[16] T. Kamae, J. Tamura and Z.-Y. Wen, Hankel determinants for the Fibonacci word and Padé approximation, Acta Arithmetica. 89 (2) (1999), 123-161.

[17] O. Salon, Suites automatiques à multi-indices, in: Séminaire de Théorie des Nombres de Bordeaux, Exposé 4, 1986-1987, 4-01-4-27; followed by an appendix by J. Shallit, 4-29A-4-36A.

[18] O. Salon, Suites automatiques à multi-indices et algébricité, C. R. Acad. Sci. Paris, Ser. I. 305 (1987), 501-504.

[19] Z.-X. Wen and W. Wu, Hankel determinants of the Cantor sequence (Chinese), Scientia Sinica Mathematica. 44 (2014): 1059-1072. 